\title{
SELF-INTERSTITIAL CLUSTERS IN SILICON
}

\author{
B. J. Coomer, J. P. Goss, A. Resende and R. Jones \\ School of Physics, The University of Exeter, Exeter EX4 4QL, United Kingdom \\ S. Öberg \\ Department of Mathematics, University of Luleä, Luleä, S95 187, Sweden \\ P. R. Briddon \\ Department of Physics, The University of Newcastle upon Tyne \\ Newcastle upon Tyne NE1 7RU, United Kingdom
}

\begin{abstract}
Local density functional calculations on the isolated silicon interstitial, $I_{1}$ and its aggregates, $I_{2}, I_{3}$ and $I_{4}$ have been performed. Several geometries are considered for each aggregate and we report preliminary results on their energetically favourable structures. The properties of the low energy structures are calculated and compared with experiment.
\end{abstract}

\section{Introduction}

The interstitial in silicon still holds many secrets. Even though interstitials are known to form extended defects [1] there is little understanding of the processes leading to their formation. Experimentally there have been surprisingly few firm assignments of any interstitial-related centre.

The $W$ optical-line (see for example Ref. [2]), seen in photoluminescence and absorption, is one example of an optical centre possibly due to an interstitial aggregate. This line, arising from a trigonal defect, is observed following crystal damage through irradiation, implantation or plasma treatment, followed heat treatment at $\sim 200^{\circ} \mathrm{C}$. Its existence is not dependent on the presence of any impurity. Carbon and oxygen dopants affect the formation of the $W$-centres since they act as sinks for interstitials and vacancies respectively. The behaviour of the line as a function of these impurity concentrations provides evidence that the defect giving rise to the $W$-line is due to interstitials [3]. This assignment is supported by the weak effect of stress on the $W$-line, which suggests that the defect cannot be vacancy related, although recent theoretical investigations have favoured a divacancy model [4]. We show that a stable, fully coordinated tri-interstitial centre exists which possesses trigonal symmetry and could account for the observed optical band.

At least one electron paramagnetic resonance (EPR) centre has been attributed to a di-interstitial aggregate [5]. The signal, labelled $P 6$ is observed following room- 
temperature radiation and displays $C_{2}$ symmetry for temperatures below $200 \mathrm{~K}$. Above this temperature the defect undergoes a thermal reorientation process so that the effective symmetry is observed to be type-II rhombic $\left(D_{2}\right.$ or $\left.D_{2 d}\right)$. The proposed model involves two interstitials, aligned along [001], either side of an occupied Si lattice site. The structure has not, to our knowledge, been tested using ab initio theory.

A model has also been proposed for a quadra-interstitial defect [6]. Tight-binding calculations show that four self-interstitials undergo a reconstruction resulting in fully coordinated defect, giving rise to an electrically and magnetically inactive centre. This would explain why this $I_{4}$-complex has not been observed experimentally.

\section{Method}

Large, hydrogen terminated, atom centred clusters $\left(\mathrm{Si}_{181+n} \mathrm{H}_{116}\right)$ are used to model the interstitial defects $I_{n}$. A first principles LDF method (AIMPRO) was used to examine their structure, electronic activity and total energies [7]. The total energies of clusters containing different models of each aggregate were compared so that the most favourable structures for $I_{1}, I_{2}, I_{3}$ and $I_{4}$ could be found. For some of the low energy defects the acceptor and donor levels were calculated using a technique described elsewhere [8]. This method typically results in an error of around $0.2 \mathrm{eV}$ for these levels.

\section{Results}

\section{The isolated interstitial: $I_{1}$}

Four forms of the isolated neutral interstitial were compared. The lowest energy form was found to be the $\langle 110\rangle$ dumbbell structure (see Fig. 1) in agreement with previous work [9]. The $\langle 100\rangle$ split-interstitial was found to be unstable against a relaxation to the $\langle 110\rangle$ form. An interstitial Si atom at a $T_{d}$ site was found to be $0.8 \mathrm{eV}$ higher in energy than the $\langle 100\rangle$ split-interstitial. A novel structure of the single interstitial was also optimised. This geometric configuration is unusual due to its trigonal symmetry $\left(C_{3}\right)$. The structure is formed by replacing a $\mathrm{Si}$ dimer by three atoms in a (111) plane which form an equilateral triangle centred equidistant from the displaced atoms. This defect was calculated to be meta-stable with an energy $2.7 \mathrm{eV}$ above that of the $\langle 110\rangle$ defect.

A filled doublet level is found deep in the band gap in agreement with others [10, 11]. A singlet level lies unoccupied in the upper half of the gap. The $\langle 110\rangle$ defect was found to possess an acceptor level at $E_{c}-0.25$. The donor level was found to lie in the valence band. The error associated with the calculated electronic levels may be rather large due to the expected large structural change concomitant with a change of charge state for the isolated interstitial [12]. 

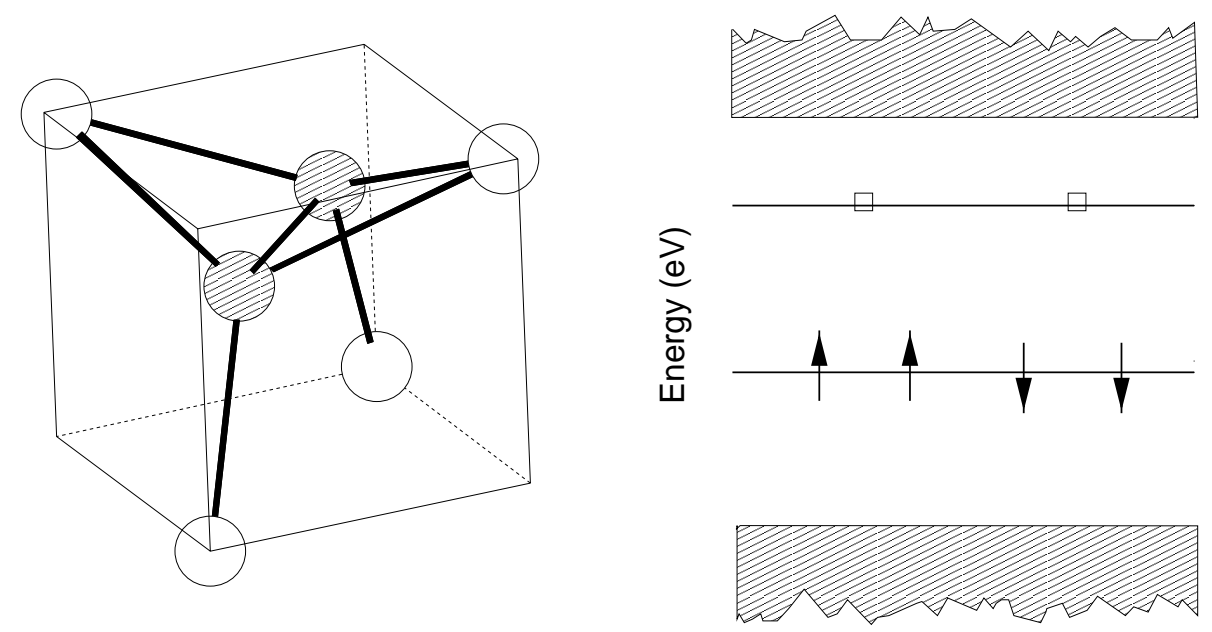

Fig. 1. Schematic of the $\langle 110\rangle$ single self-interstitial configuration and Kohn-Sham levels. The shaded circles represent the two atoms sharing the single lattice site.

\section{The di-interstitial: $I_{2}$}

Five forms of the neutral di-interstitial were considered in this study. The lowest energy model is calculated to be a $\langle 110\rangle$ structure centred at a $H$-site (see Fig. 2A). This structure may be considered to be a fragment of an interstitial chain inserted into a $\langle 110\rangle$ channel [1] and will be referred to as the $I_{2}$ chain fragment. The optimised models will be discussed in reverse order of energy and energies are given relative to the optimised $I_{2}$ chain fragment energy, $E_{c h}$.

The model assigned by Lee [5] to the EPR centre labelled $P 6$ was found to have energy $E_{c h}+2.90 \mathrm{eV}$ with the symmetry constrained to $D_{2 d}$. The relaxation of the symmetry constraint results in an energy lowering of $1.1 \mathrm{eV}$. However the resultant structure has little in common with the ground state structure proposed by Lee. The relaxation results in the high symmetry defect spontaneously dissociating into two neighbouring interstitials with no recognisable symmetry.

The single $\langle 100\rangle$ split-interstitial with an additional neighbouring $T_{d}$ interstitial along the principal [100] direction was also optimised and found to have energy, $E_{c h}+2.67 \mathrm{eV}$.

Two structures containing $\langle 100\rangle$ split-interstitial pairs were optimised. The nearest neighbour model consists of two neighbouring $\langle 100\rangle$ interstitials resembling the nitrogen defect, $\left(N_{i}\right)_{2}$ [13]. The relaxed structure was found to possess $C_{2 h}$ symmetry and have energy, $E_{c h}+0.41 \mathrm{eV}$. Moving the pair to next-nearest neighbour position reduces this by $0.15 \mathrm{eV}$ and results in a defect with $C_{2 v}$ symmetry (Fig. 2B). The two 3 -fold coordinated atoms (shown shaded in Fig. 2B) lie coplanar with their bonded neighbours.

A further, small reduction in energy is produced by relaxing the planar symmetry constraint on the next-nearest neighbour model (Fig. 2B). The two 3-fold coordinated atoms move along $\langle 100\rangle$ in opposite directions. This distorted structure has $C_{2}$ symmetry. We expect, however, that thermal averaging effects would cause 


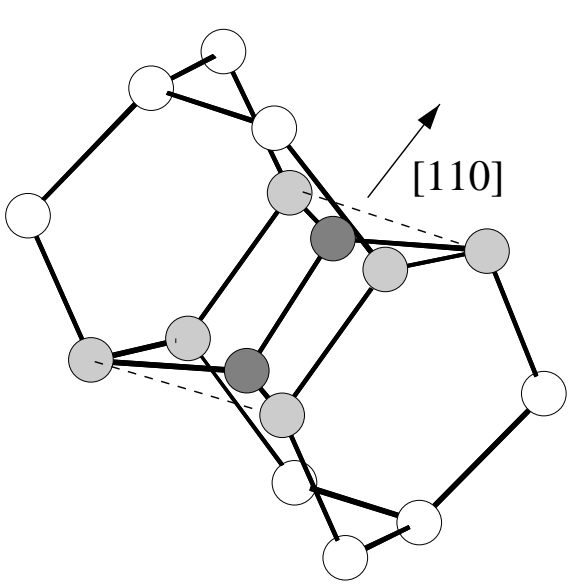

A

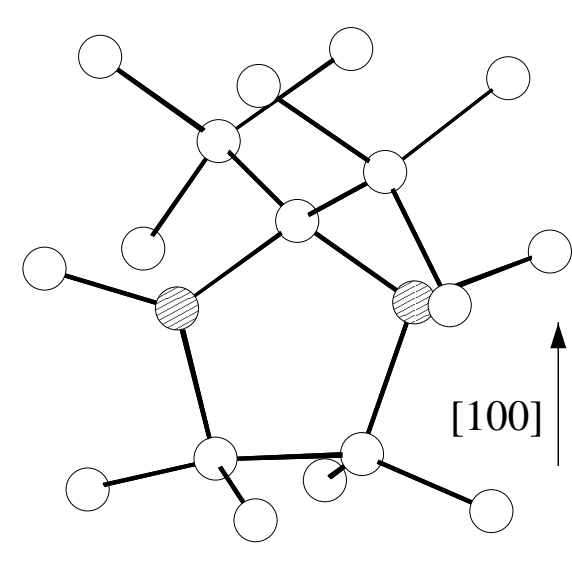

B

Fig. 2. Two possible forms for the di-interstitial in silicon. A: A two-interstitial segment taken from the $\langle 110\rangle$ chain centred at a $H$-site. The two interstitials (dark shaded) interupt two Si-Si bonds (dotted lines). The hexagonal ring is shown shaded (light) to aid the eye. B: The next-nearest neighbour $\langle 100\rangle$ di-split-interstitial.

the centre to be observed in the higher symmetry configuration at reasonably low temperatures.

The lowest energy structure calculated was the $\langle 110\rangle$ chain segment (Fig. 2A). This was found to possess $C_{i}$ symmetry. However, the buckled nature of the interstitial dimer (shaded) would probably not be apparent above room temperature. Thermal averaging would introduce a principal $C_{2}$ axis pointing along $\langle 110\rangle$, as indicated in Fig. 2, resulting in overall $C_{2 h}$ symmetry for the centre.

\section{The neutral tri-interstitial: $I_{3}$}

Two models have thus far been compared. The lower energy structure was found to possess trigonal symmetry $\left(C_{3 v}\right)$ with the $C_{3}$ axis along $\langle 111\rangle$ (see Fig. 3 ). The energy of this structure was compared with that of a modified Takeda $I_{4}$ structure [6]. One atom was removed from this $I_{4}$ defect and the resultant structure was relaxed. This was found to be $1.1 \mathrm{eV}$ higher in energy than the $C_{3 v}$ tri-interstitial.

The trigonal tri-interstitial defect can be created by inserting three Si atoms into the centres of three neighbouring bonds parallel to (111) as shown in Fig. 3. These atoms displace the three atoms lying above them away compressing the bond between atoms marked 1, 2 and opening up the hexagon at the base of the figure.

Since all bonds are fully saturated, there are no midgap levels. The calculated levels indicate some filled states around $0.2 \mathrm{eV}$ above $E_{v}$ and possibly some shallow states below $E_{c}$. The centre has $C_{3 v}$ symmetry and we tentatively identify it with the $W$-centre.

This optical defect gives a sharp optical line at $1.018 \mathrm{eV}$ arising from a $A_{1} \rightarrow A_{1}$ internal transition with a lifetime of the order of a few nanoseconds. There are $A_{1}$ 


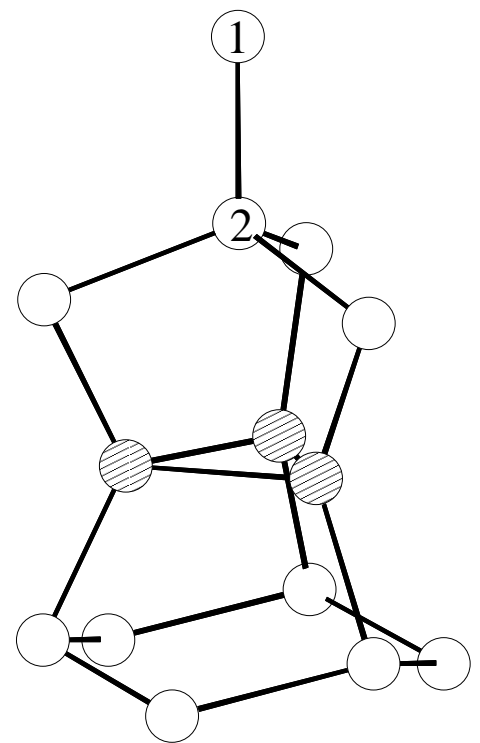

A

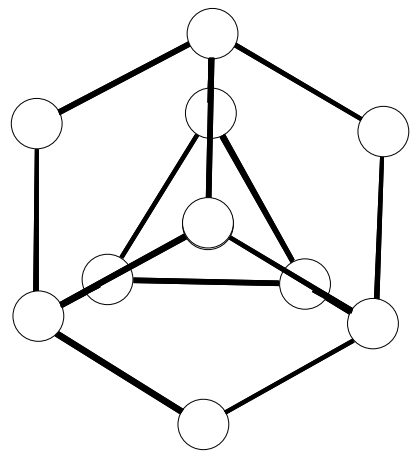

B

Fig. 3. Structure of the low energy tri-interstitial defect. A: Three interstitials are added (shaded) interrupting three $\mathrm{Si}-\mathrm{Si}$ bonds to form the defect. The centre of the triangle lies approximately at the $T_{d}$ interstitial-site. B: The view along the principal $\langle 111\rangle$ direction. The bond formation between the interstitials results in trigonal symmetry.

and $E$ excited states lying about $30 \mathrm{meV}$ higher in energy and the defect has a $A_{1}$ local vibrational mode at $564.2 \mathrm{~cm}^{-1}(70.0 \mathrm{meV})$ which shifts $16 \mathrm{~cm}^{-1}$ when one of the core atoms is replaced by ${ }^{30} \mathrm{Si}$. Such a large shift can only be expected from a unique $\mathrm{Si}$ atom which must then lie on the $C_{3}$ axis. $W$-centres are created by heavy ion or neutron irradiation followed by heat treatment at $250^{\circ} \mathrm{C}$. The concentration of $W$-centres appears to rapidly saturate with dose and the defects anneals out at about $327^{\circ} \mathrm{C}$. These remarks assume that harsher radiation treatments or higher temperature anneals do not introduce non-radiative centres which compete with the $W$-luminescence. Unfortunately, the centres have not been studied in absorption. Bombardment with inert-gas ions produces a family of lines believed to be due to a inert gas atom trapped near the defect.

The model in Fig. 3 is consistent with these observations. States at around $E_{v}+0.25$ are localised on the ring of three $\mathrm{Si}$ atoms and could participate in the luminescence. The short bond between atoms 1 and 2 is expected to give a local vibrational mode above the Raman frequency with a pronounced Si isotopic shift on two Si atoms. The enlarged hexagon would provide space for both inert gas atoms. Two hydrogen atoms will bind to two of the interstitials breaking one of the bonds of the triangle. We have found that this action removes gap states and hence optical activity. This is consistent with reports of the passivation of the $W$-centre[4]. Further studies are necessary to confirm the model. 


\section{The quadra-interstitial: $I_{4}$}

Only one model has been considered so far. The model suggested by Takeda et al. [6] was relaxed. The optimised structure (Fig. 4) compares well with that calculated by Takeda. All atoms are 4-fold coordinated. The electrical activity remains to be investigated.
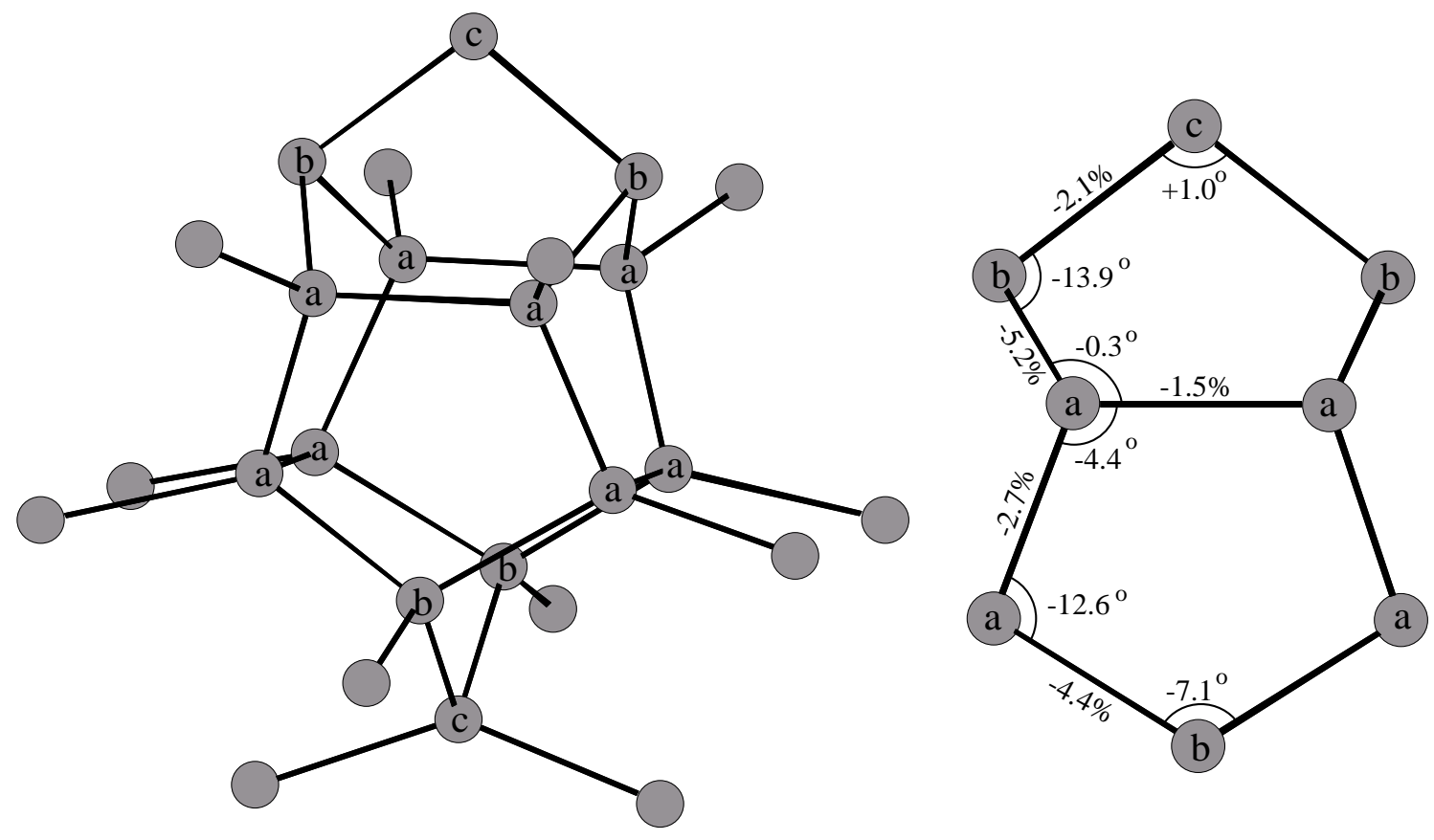

Fig. 4. Optimised quadra-interstitial model. Distortions from the ideal bond lengths and angles are shown.

\section{Conclusions}

The lowest energy form of the isolated interstitial is calculated to be the $\langle 110\rangle$ splitinterstitial in agreement with others. It is possibly an acceptor with a $(-/ 0)$ level at around $E_{c}-0.2 \mathrm{eV}$. The di-interstitial has two low energy forms - within $0.26 \mathrm{eV}$ of each other. The lower energy structure is the $\langle 110\rangle$ chain segment centred at the $H$-site. The electrical activity of these centres remains to be investigated. The diinterstitial model proposed by Lee was found to be unstable. This casts doubt on their assignment to the P6 EPR centre.

An interesting model for $I_{3}$ was found. Its trigonal nature makes it an excellent candidate as a model for the $W$-centre. Further investigations are underway. The structure proposed by Takeda et al. [6] for $I_{4}$ was found to be stable and its optimised structure agrees with that calculated using tight-binding methods.

\section{References}

[1] J. Kim, J. W. Wilkins, F. S. Khan, and A. Canning, Phys. Rev. B 55, 16186 (1997). 
[2] G. Davies, E. C. Lightowlers, and Z. E. Ciechanowskia, J. Phys. C: Solid State Phys. 20, 191 (1987).

[3] M. Nakamura, S. Nagia, Y. Aoki, and H. Naramoto, Appl. Phys. Lett. 72, 1347 (1998).

[4] S. K. Estreicher, J. Weber, A. Derecskeikovacs, and D. S. Marynick, Phys. Rev. B 55, 5037 (1997).

[5] Y. H. Lee, Appl. Phys. Lett. 73, 1119 (1998).

[6] N. Aria and S. Takeda, Phys. Rev. Lett. 78, 4265 (1997).

[7] R. Jones and P. R. Briddon, in Identification of Defects in Semiconductors, Vol. 51A of Semiconductors and Semimetals, edited by M. Stavola (Academic Press, Boston, 1998), Chap. 6, p. 287.

[8] A. Resende and R. Jones, Phys. Rev. Lett. 82, 2111 (1999).

[9] S. J. Clark and G. J. Ackland, Phys. Rev. B 56, 47 (1997).

[10] D. J. Chadi, Phys. Rev. B 46, 9400 (1992).

[11] P. W. Leary, Ph.D. thesis, University of Exeter, 1997.

[12] Y. B. Yam and J. D. Joannopolous, Phys. Rev. B 30, 2216 (1984).

[13] R. Jones, S. Öberg, F. Berg Rasmussen, and B. Bech Nielsen, Phys. Rev. Lett. 72, 1882 (1994). 